\title{
"See the Sea" Information System. Current Capabilities and Prospects of Development
}

\author{
Olga Yu. Lavrova, Evgeny A. Loupian, Marina I. Mityagina, Ivan A. Uvarov \\ Space Research Institute, Russian Academy of Sciences, Moscow, Russia \\ olavrova@iki.rssi.ru
}

\begin{abstract}
The information system "See the Sea" was developed in IKI to provide researchers in the field of oceanology with access to satellite, meteorological and other data, as well as data processing products. The system provides not only access to long-term data archives, but also to on-line data analysis tools, such as brightness correction and image classification, analysis of spectral characteristics of objects. The specialized subsystem of the description of the discovered phenomena is aimed at joining, accumulation, and systematization of the knowledge gained as the working result of researchers team. The taxonomic classifier has been created to describe the phenomena that can be detected on the sea surface. Further development of the system involves the integration of new data such as Argo buoy measurements and satellite altimetry, as well as the development of new data analysis tools.
\end{abstract}

Keywords: remote sensing, monitoring, World Ocean, radar imagery, hyperspectrometers, sea surface 


\title{
ИНФОРМАЦИОННАЯ СИСТЕМА SEЕ THЕ SEA: ТЕКУЩИЕ ВОЗМОЖНОСТИ И ПЕРСПЕКТИВЫ РАЗВИТИЯ
}

\author{
О.Ю. Лаврова, Е.А. Лупян, М.И. Митягина, И.А. Уваров \\ Институт космических исследований РАН, Москва, Россия \\ olavrova@iki.rssi.ru
}

\begin{abstract}
Информационная система See the Sea разработана в ИКИ РАН для обеспечения исследователей в области океанологии возможностями доступа к спутниковым, метеорологическим и другим данным, а также результатам обработки данных. Система обеспечивает не только доступ к многолетним архивам данных, но и к инструментам анализа данных on-line, таким как яркостная коррекция и классификация изображений, анализ спектральных характеристик объектов. Специализированная подсистема описания обнаруженных явлений призвана накапливать, объединять и систематизировать знания, полученные в результате работы группы исследователей. Для описания явлений, которые могут быть обнаружены на морской поверхности, создан таксономический классификатор. Дальнейшее развитие системы сопряжено с добавлением новых данных, таких как измерения с буев Argo и спутниковая альтиметрия, а также разработкой новых инструментов анализа.
\end{abstract}

Ключевые слова: дистанционное зондирование, мониторинг, Мировой океан, радиолокационные изображения, гиперспектрометры, морская поверхность

\section{Введение}

Основным назначением информационной системы See The Sea (STS), разработанной в Институте космических исследований РАН в 2011 г., является обеспечение исследователей возможностями доступа и инструментами анализа информации, полученной на основе данных спутниковых наблюдений (как оперативных, так и из накопленных архивов), для изучения различных процессов происходящих в океане и атмосфере над ним [1, 2]. Информационная система STS использует инфраструктуру и архивы данных, накопленные в Центре коллективного пользования «ИКИ-Мониторинг» [3].

Изучение океанологических процессов и явлений, определение параметров течений, выносов рек, нефтяных загрязнений, ледовой обстановки и многое другое, требует проведение комплексного анализа всех доступных спутниковых данных совместно с гидрометеорологической информацией. Необходима возможность описания различных процессов и явлений, происходящих в Мировом океане, и ведения долговременных баз данных таких описаний. Требуется специализированный инструментарий, возможность сохранения 
результатов анализа и обработки и использование их в дальнейших исследованиях, построение различных информационных продуктов.

В работе кратко представлены основные возможности системы, используемые данные, и перспективы развития.

\section{Виды данных, используемых в системе}

Система STS обеспечивает доступ к постоянно пополняемым архивам спутниковых, метеорологических и тематических данных. Рассмотрим основные их виды.

Среди спутниковых данных радиолокационные изображения являются основным источником для наблюдения морской поверхности. Накоплено около 5000 сцен приборов ERS1/2 SAR и Envisat ASAR за период 1995 -2012 гг. Преимущественно имеющиеся изображения покрывают Черное, Балтийское и Каспийское моря. В настоящее время постоянно пополняется архив данных со спутников Sentinel -1А и -1B.

Данные оптического диапазона различаются по пространственному разрешению и спектральным диапазонам. Данные среднего разрешения, полученные приборами NOAA AVHRR, Terra/Aqua MODIS, Envisat MERIS, могут использоваться для определения различных характеристик морской поверхности, таких как цвет и температура воды, ледовый покров. Архив таких данных ведется с 2000 года. Данные высокого разрешения, полученные приборами со спутников Landsat 5/7/8, а также Sentinel -2A, -2B, обеспечивают наиболее детальный анализ наблюдаемых явлений на поверхности моря.

Специализированная аппаратура на борту спутника Sentinel 3A нацелена на получение информационных продуктов для определения характеристик водных объектов, прежде всего, цвета и температуры: OLCI (Ocean and Land Colour Instrument), SLSTR (Sea and Land Surface Temperature Radiometer).

Особую роль играют гиперспектральные приборы. Они позволяют различать типы водной поверхности благодаря наиболее подробной передаче спектральных характеристик наблюдаемого объекта. Такие данные были получены приборами Hyperion и HІСО (съемка прибором НICO проводилась по заказу ИКИ РАН в рамках совместного договора между ИКИ PAH и Naval Research Lab) [4]

Метеорологические данные интегрированы в систему для совместного анализа со спутниковыми изображениями при исследовании происходящих процессов. Предусмотрена работа с такими величинами, как температура воздуха, количество осадков, относительная влажность, атмосферное давление, облачность, нисходящие и восходящие потоки солнечной радиации, скорость и направление ветра. Данные получены на основе модели NCEP Climate Forecast System Version 2 (https://rda.ucar.edu/datasets/ds094.0/) [5]

Географическая основа включает ряд векторных слоев, используемых при визуализации данных. В их числе береговая линия, карты глубин, гидрография суши.

Данные буев системы Argo включают измерения температуры и солености воды. В системе STS проводилась интеграция и визуализация данных о положении буев. Предполагается расширение функциональности для работы с этими данными.

STS -открытая система. Предусмотрена возможность интегрирования в систему открытых данных новых сенсоров вновь запускаемых спутников

\section{Возможности работы с данными}

Картографический интерфейс системы See the Sea основан на разработанной в ИКИ РАН технологии GEOSMIS. [6] Это обеспечивает возможность доступа ко всем видам 
интегрированных в систему данных, выбору параметров визуализации и управлению инструментами анализа данных.

Поиск данных является отправной точкой исследования и подчиняется множеству критериев: качественных, временных, пространственных. Данные сгруппированы в разделах интерфейса ("вкладках") по типам. Поиск спутниковых данных учитывает такие параметры, как наличие тематических продуктов обработки, количество облачности (для оптических данных), поляризация (для радиолокационных).

Подготовка спутниковых данных к работе является неотъемлемым этапом исследования. Это связано с тем, что возможность обнаружения тех или иных явлений во многом зависит от параметров визуализации. Применительно к радиолокационным данным, имеющим, по своей природе, большой перепад величин измеренной интенсивности сигнала, подбор подходящей передаточной функции принципиально важен для контрастного отображения наблюдаемого явления. Также предусмотрено использование цветового синтеза поляризаций. Настройка параметров визуализации актуальна и для изображений оптического диапазона. Выбор информативных спектральных каналов и яркостная коррекция позволяют подготовить изображение для наблюдения явлений. Особенно можно отметить возможность цветового синтеза разных изображений, будь то разновременные изображения или снимки, полученные разными системами.

Основным специализированным исследовательским инструментом системы See the Sea является подсистема описания явлений. Она призвана накапливать, объединять и систематизировать знания, полученные в результате работы группы исследователей. Для описания явлений, которые могут быть обнаружены на морской поверхности, создан таксономический классификатор. Обнаружение исследователем явления предусматривает добавление записи в базу данных. При этом происходят следующие операции с данными:

- участок обнаружения явления регистрируется как графический элемент, на карту наносится контур распространения явления;

- явлению присваиваются атрибуты в соответствии с классификатором;

- определяются количественные характеристики объекта;

- изображение, на котором обнаружено явление, маркируется в соответствии с классом явления;

- с изображением ассоциируется его общее описание.

Такой подход к организации работы позволяет впоследствии выполнять поиск по базе данных зарегистрированных явлений определенного типа в заданном регионе и промежутке времени, проводить статистическую обработку, строить карты пространственного распределения зарегистрированных явлений. Пример такой карты, на которой представлены выявленные нефтяные загрязнения за период с 2009 по 2012 год в акватории Черного моря, приведен на рисунке ниже. По каждому зарегистрированному явлению можно также получить различную информацию, входящую в его описание, в том числе, исходные спутниковые данных, на которых были обнаружены явления заданного типа.

В системе реализована возможность классификации изображений на основе их спектральнояркостных характеристик: в режимах как обучаемой, так и необучаемой классификации. Это позволяет автоматизированным образом получать карты распространения поверхностных явлений, карты разных типов водной поверхности.

Специализированный инструмент реализован для работы с гиперспектральными данными. Для заданных объектов наблюдения на водной поверхности, а также для групп таких объектов, возможно построение спектральных профилей в виде графиков. Это позволяет наглядно определить характерные особенности спектральных характеристик качественно различающихся типов поверхности и, таким образом, выявить наиболее информативные участки 
электромагнитного спектра. Информативные каналы гиперспектрометра впоследствии могут быть использованы для проведения классификации и построения карт.

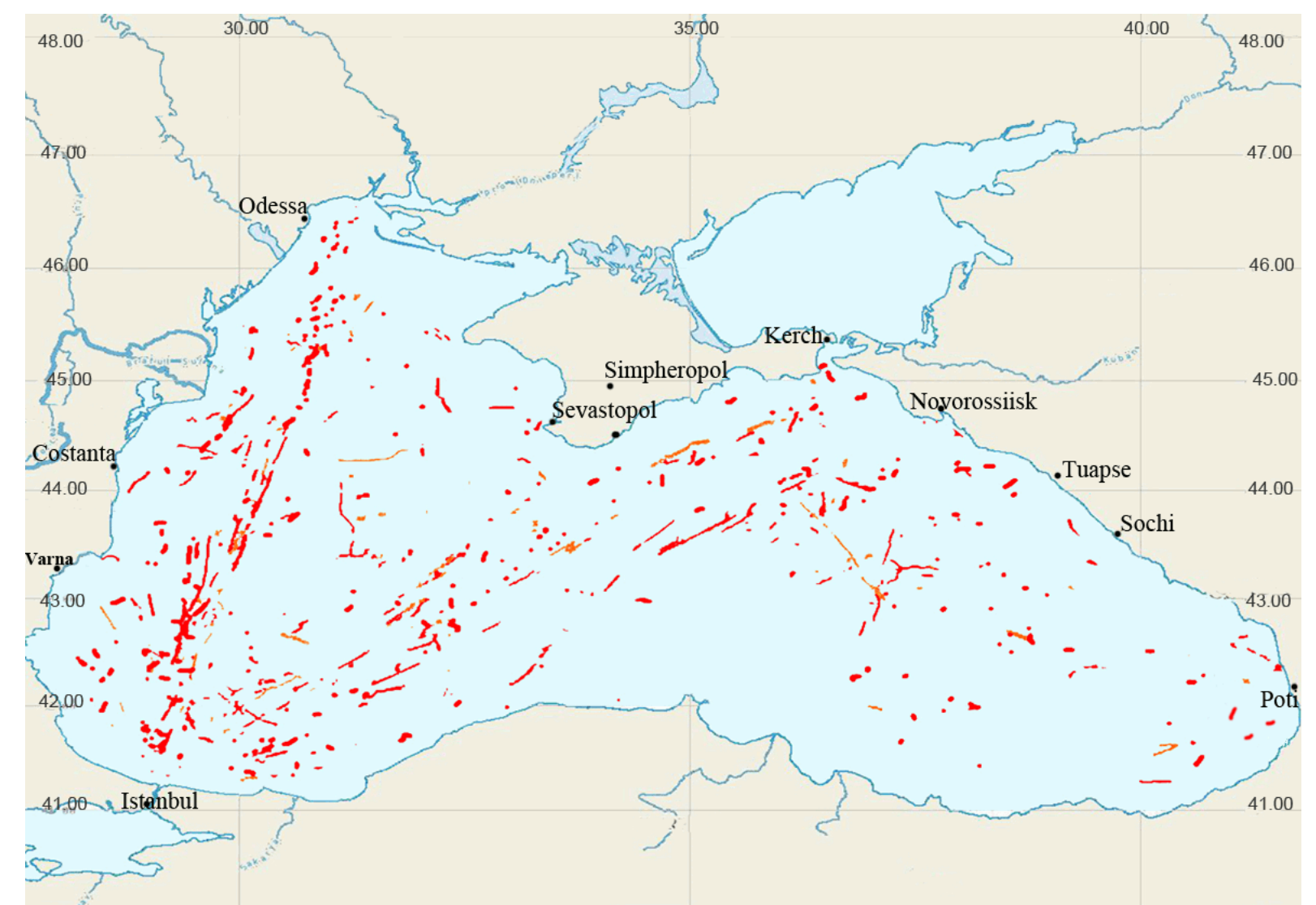

Карта распределения нефтяных загрязнений в Черном море за период с 2009 по 2012 год [7]

\section{Перспективы развития}

Дальнейшее развитие STS связано с увеличением количества видов данных, интегрированных в систему и разработкой новых инструментов анализа. В частности, планируется внедрение средств работы с данными подспутниковых измерений для обеспечения их совместного анализа со спутниковыми данными.

Планируется расширение возможностей работы с данными буев Argo. Будут организованы как оперативный сбор данных о состоянии, так и накопление в виде многомерного массива истории измерений в разных частях акваторий на поверхности и на глубине для последующей визуализации в виде карт и графиков.

Ожидается появление новых возможностей изучения явлений на морской поверхности благодаря интеграции STS данных спутниковой альтиметрии и инструментария для работы с этими данными. Предполагается внедрение в STS численных моделей, позволяющих определять количественные характеристики состояния морской поверхности на основе спутниковых данных.

\section{Заключение}

В современных условиях работа с многолетними архивами спутниковых данных, изучение пространственно-временной изменчивости, выявление трендов и построение прогнозов и т.п. невозможно без использования специализированных информационных систем, таких как STS. Оперативная оценка состояния морской среды: определение параметров течений, выносов рек, 
нефтяных загрязнений, ледовой обстановки и многое другое, требует проведение комплексного анализа всех доступных спутниковых данных совместно с гидрометеорологической информацией [8-10]. Использование такой системы необходимо для оперативной работы с изображениями большого размера (несколько гигабайт) и совместного анализа разнородных данных. Опыт использования See the Sea указывает на то, что система является необходимым инструментом исследования.

Развитие информационной системы STS осуществляется в рамках темы «Мониторинг» (госрегистрация № 01.20.0.2.00164) с использованием возможностей Центра коллективного пользования «ИКИ-мониторинг» [3].

Работы по изучению различных процессов в морских акваториях проводятся с использованием информационной системы STS в рамках различных грантов. В частности, изучение гидродинамических процессов, связанных с распространением речных и лагунных вод в море проводятся при финансовой поддержке Российского фонда фундаментальных исследований в рамках гранта №17-05-00715.

\section{References}

[1] Loupian E.A., Matveev A.M., Uvarov I.A., Bocharova T. Yu., Lavrova O. Yu., Mityagina M.I. The Satellite Service See the Sea - a tool for the Study of Oceanic Phenomena and Processes, Sovremennye Problemy Distantsionnogo Zondirovaniya Zemli iz Kosmosa, 2012, V. 9, No 2, pp. 251-262. (In Russian)

[2] Loupian E., Lavrova O., Kashnizky A., Uvarov I. "See The Sea" - new opportunities for distributed collaboration aimed at solution of oceanographic problems using remote sensing, Bollettino di Geofisica Teorica ed Applicata, 2018, Vol. 59, Supl. 1, pp. 91-93.

[3] Loupian E.A., Proshin A.A., Burtsev M.A., Balashov I.V., Bartalev S.A., Efremov V.Yu., Kashnitskiy A.V., Mazurov A.A., Matveev A.M., Sudneva O.A., Sychugov I.G., Tolpin V.A., Uvarov I.A. IKI center for collective use of satellite data archiving, processing and analysis systems aimed at solving the problems of environmental study and monitoring, Sovremennye Problemy Distantsionnogo Zondirovaniya Zemli iz Kosmosa, 2015, 12(5), pp. 263-284 (In Russian).

[4] Mityagina M.I., Lavrova O. Yu., Uvarov I.A. "See the Sea": Multi-user information system for investigating processes and phenomena in coastal zones via satellite remotely sensed data, particularly hyperspectral data // Remote Sensing of the Osean, Sea Ice, Coastal Waters, and Large WaterRegions Location, Book Series: Proceedings of SPIE, Netherlands, Amsterdam, Article number: 92401C, 2014. Vol.9240. DOI: 10.1117/12.2067300.

[5] Uvarov I.A., Khalikova O.A., Balashov I.V., Bourtsev M.A., Loupian E.A., Matveev A.M., Platonov A.E., Proshin A.A., Tolpin V.A., Krasheninnikova Yu. S. Meteorological data management in framework of the satellite monitoring information systems // Sovremennye Problemy Distantsionnogo Zondirovaniya Zemli iz Kosmosa. 2013. Vol.10. № 2. P. 30-45. (In Russian)

[6] Tolpin V.A., Balashov I.V., Efremov V. Yu., Loupian E.A., Proshin A.A., Uvarov I.A., Flitman E.V. The GEOSMIS System: Developing Interfaces to Operate Data in Modern Remote Monitoring Systems // Sovremennye Problemy Distantsionnogo Zondirovaniya Zemli iz Kosmosa. 2011. V. 8. № 3. P. 93-108. (In Russian)

[7] Mityagina, M., Lavrova, O. Satellite Survey of Inner Seas: Oil Pollution in the Black and Caspian Seas, Remote Sensing, 2016, 8, 875. DOI:10.3390/rs8100875.

[8] Lavrova O., Mityagina M. Satellite Survey of Internal Waves in the Black and Caspian Seas, Remote Sensing, 2017, 9, 892. DOI: 10.3390/rs9090892. 
[9] Lavrova O.Yu., Soloviev D.M., Strochkov M.A., Bocharova T.Yu., Kashnitsky A.V. River plumes investigation using Sentinel-2A MSI and Landsat-8 OLI data, Remote Sensing of the Ocean, Sea Ice, Coastal Waters, and Large Water Regions, 2016, Vol. 9999, 99990G. DOI: 10.1117/12.2241312.

[10] Lavrova O.Yu., Mityagina M.I., Bocharova T.Yu., Kostianoy A.G. Long-term monitoring of sea ice conditions in the Kerch Strait by remote sensing data, Proc. SPIE 10422, Remote Sensing of the Ocean, Sea Ice, Coastal Waters, and Large Water Regions, 2017, 104220L. doi:10.1117/12.2277829. 